\section{On the role of bias in dissociated phonological priming effects: A reply to Goldinger (1999)}

\author{
MARYBETH HAMBURGER \\ Georgia State University, Atlanta, Georgia \\ and \\ LOUISA M. SLOWIACZEK \\ Bowdoin College, Brunswick, Maine
}

\begin{abstract}
Phonological priming studies have revealed two dissociated effects: low-similarity facilitation and highsimilarity interference (Hamburger \& Slowiaczek, 1996; Slowiaczek \& Hamburger, 1992). Because these two effects are influenced by different variables, they most likely reflect different processes that occur during auditory word recognition. Goldinger (1999) suggests that one bias is responsible for all phonological priming effects. In this reply, we argue against such a position. Although low similarity facilitation is likely the product of this bias, the data on phonological priming indicate that the dissociated highsimilarity interference cannot be produced by the same mechanism. Instead, the data indicate that high-similarity interference may reflect lexical processes.
\end{abstract}

Research on phonological priming promises to reveal a subset of those mechanisms operating during auditory word recognition. However, as Goldinger (1999) indicates, priming effects reflect top-down, contextually driven processes. Therefore, phonological priming data must be closely examined to distinguish between processes that occur automatically during auditory word recognition (regardless of whether priming is used to examine them) and those that result from the contrived situation of a priming experiment. Goldinger suggests that one method of distinguishing between separate processes is to set those processes in opposition to each other (e.g., facilitatory bias and inhibitory lexical effects). Another method is to look for variables that differentially influence dissociated priming effects. Both methods have revealed two distinct phonological priming effects: lowsimilarity facilitation and high-similarity interference (Slowiaczek \& Hamburger, 1992). Goldinger proposes that a single bias is responsible for all phonological priming. We agree with Goldinger that low-similarity facilitation is a strategic effect. However, because highsimilarity interference is dissociated from low-similarity facilitation, the same bias cannot be responsible for both effects. Instead, we maintain that high-similarity interference reflects lexical competition.

Correspondence concerning this article should be sent to L. M. Slowiaczek, Department of Psychology, Bowdoin College, 6900 College Station, Brunswick, ME 04011 (e-mail: lslowiac@polar.bowdoin.edu).

\section{Two Dissociated Phonological Priming Effects}

Two phonological priming effects were first distinguished by Slowiaczek and Hamburger (1992). They found that response times (RTs) to auditory target words preceded by primes sharing one or two initial phonemes with the target are faster than RTs to targets preceded by unrelated primes (i.e., low-similarity facilitation). Slowiaczek and Hamburger also found that RTs increase as the phonological overlap between primes and targets increases from one or two phonemes to three phonemes (i.e., highsimilarity interference). Moreover, whenever low-similarity facilitation is not present, high-similarity interference leads to inhibitory priming (i.e., slower responses to targets preceded by primes sharing three initial phonemes than to targets preceded by unrelated primes). Goldinger (1999) suggests that a single bias is responsible for both effects. However, these two effects most likely reflect separate processes in auditory word recognition, because the two priming effects are differentially influenced by several variables.

Slowiaczek and Hamburger (1992) identified three variables that dissociate the two phonological priming effects: (1) lexicality of the prime-word or nonword; (2) modality of prime presentation - auditory or visual; and (3) presence or absence of identity priming trials. Specifically, prime lexicality influences high-similarity interference but does not influence low-similarity facilitation. They found that equal amounts of low-similarity facilitation are revealed for word and nonword primes but that high-similarity interference is revealed with word primes only. Prime modality, on the other hand, influences low-similarity facilitation but does not influence high-similarity interference. Equal amounts of highsimilarity interference are revealed for auditory and visual primes, but low-similarity facilitation is revealed for auditory primes only. Moreover, prime modality affects low-similarity facilitation only if identity priming trials are not presented during the experiment. Only when identity priming trials are included in the experiment do both auditorily and visually presented primes produce low-similarity facilitation. Slowiaczek and Hamburger observed no effect of the presence or absence of identity priming trials on high-similarity interference. On the basis of this pattern of results, Slowiaczek and Hamburger concluded that low-similarity facilitation is a perceptual, prelexical effect due to auditory similarity between primes and targets, whereas high-similarity interference is a lexical effect due to competition between lexical representations of primes and targets.

\section{Expectancy and Phonological Priming}

Expectancy condition is a fourth variable that has recently been found to distinguish between high-similarity interference and low-similarity facilitation (Goldinger, 1999; Hamburger \& Slowiaczek, 1996). Goldinger (1999) 
and Hamburger and Slowiaczek conducted studies in which phonological priming was examined under conditions that promote strategic processing (high-expectancy condition) or discourage strategic processing (lowexpectancy condition). Generalizing from the semantic priming literature (Neely, 1991; Neely \& Keefe, 1989), strategic processing in phonological priming experiments is most likely under high-expectancy conditions in which the majority of trials use phonologically related primetarget pairs (a high phonological relatedness proportion or PRP) and a long interstimulus interval (ISI) is employed. Strategic processing is less likely under lowexpectancy conditions in which the majority of trials use unrelated prime-target pairs (a low PRP) and a short ISI is employed.

Under high-expectancy conditions (PRP of 50\%-75\% and 500-msec ISI), low-similarity facilitation is found (Goldinger, 1999; Goldinger, Luce, Pisoni, \& Marcario, 1992; Hamburger \& Slowiaczek, 1996). Under lowexpectancy conditions (PRP of $21 \%$ and $50-\mathrm{msec}$ ISI), low-similarity facilitation is negligible (Goldinger, 1999; Hamburger \& Slowiaczek, 1996). Moreover, low-similarity inhibition has even been observed with a PRP of $10 \%$, a 50-msec ISI, and targets embedded in noise (Goldinger et al., 1992). Because low-similarity facilitation increases as PRP and ISI increase, this effect is believed to be due to a strategy. Participants anticipate targets that have one or two initial phonemes in common with the prime, and thus respond more quickly to these items.

Whereas PRP and ISI are positively correlated with low-similarity facilitation, they are negatively correlated with high-similarity interference. Under high-expectancy conditions, high-similarity interference is encumbered by strategic facilitation and produces negligible inhibition (Hamburger \& Slowiaczek, 1996; Slowiaczek \& Hamburger, 1992). However, under low-expectancy conditions, high-similarity interference produces reliable inhibition (Hamburger \& Slowiaczek, 1996). Because Neely (1991; Neely \& Keefe, 1989) has found that strategic effects are maximized under high relatedness proportion, long ISI conditions and reduced under low relatedness proportion, short ISI conditions, Hamburger and Slowiaczek argue that high-similarity interference is most likely not a strategic effect. If it were strategic, high-similarity interference should not increase under low-expectancy conditions.

\section{Goldinger's (1999) Criticisms}

Goldinger's (1999) criticisms of Hamburger and Slowiaczek (1996) focus on several assumptions that may be inaccurate. First, Goldinger proposes that the relatively long RTs observed in the high-expectancy condition reflect an inefficient bias on the part of the participants. Because of the "erratic nature" of the experiment, Goldinger argues, participants "struggle to avoid 'false positives." That is, RTs increase because participants try not to expect targets that are phonologically similar to the prime. Such a conclusion is unsupported until the ISI is manipulated separately from the PRP. An equally reasonable explanation for slower RTs in the highexpectancy condition is the slower timing of trials in the high-expectancy condition brought about by the longer ISI (see, e.g., Stone, Joaquim, \& Gabrieli, 1995). Goldinger's support of his position (his test "using consistent levels of phonological overlap within blocks of trials") is too vague to allow reliable conclusions.

Second, Goldinger (1999) believes the conclusion that high-similarity interference is not a strategic effect is unwarranted because it relies on (1) the observation that "the three-phoneme interference effect does not vanish in the low-expectancy condition," and (2) the assumption that biases are completely eliminated in the lowexpectancy condition. Neither of these arguments is valid. With regard to the first argument, Goldinger's framing of the logic in Hamburger and Slowiaczek (1996) states that eliminating bias in an experiment should eliminate priming if the priming is based on bias. Because the inhibitory priming was not eliminated in Hamburger and Slowiaczek the effect must not be the result of bias. Thus, the logic of the experiment, as portrayed by Goldinger, relies on the finding of a null result (i.e., no priming or no change in priming). In truth, however, the critical finding of Hamburger and Slowiaczek is the statistically significant interaction between priming and expectancy. The data from Hamburger and Slowiaczek revealed that inhibition increased when biases were reduced. Why should any reduction in bias lead to greater effects if the bias is causing the effect? Goldinger also found this statistically significant interaction between priming and expectancy such that more high-similarity interference and less low-similarity facilitation are observed in the lowexpectancy condition than in the high-expectancy condition. Although Goldinger chose not to discuss the implications of this interaction, Hamburger and Slowiaczek concluded that this statistically significant interaction indicates that high-similarity interference is not the product of strategic operations brought on by the experimental situation. Furthermore, the fact that prime lexicality influences high-similarity interference suggests that this effect reflects lexical processing (Slowiaczek \& Hamburger, 1992).

With regard to Goldinger's (1999) second argument, Hamburger and Slowiaczek (1996) did not need to completely eliminate biases in the low-expectancy condition in order to reach their conclusion. Instead, it is only necessary to reduce strategic processing enough to produce reliable changes in the results. In all likelihood, some strategic processing probably takes place in nearly all priming experiments because of the top-down nature of priming. As a result, Hamburger and Slowiaczek may not have completely eliminated bias. We took the significant interaction between priming and expectancy condition, however, as evidence that strategic processing was reduced in the low-expectancy condition relative to the high- 
expectancy condition. This conclusion is not compromised by Goldinger's observation that the degree of bias in the two expectancy conditions was never directly assessed.

Finally, Goldinger (1999) criticizes Hamburger and Slowiaczek (1996) for failing to demonstrate that biases had been eliminated in the low-expectancy condition. He replicates Hamburger and Slowiaczek and assesses bias by analyzing the RTs for unrelated trials as a function of their temporal position in the experiment (a "time-window" analysis). This procedure allowed Goldinger to assess the "'cost" "(Neely, 1977; Posner \& Snyder, 1975) of strategic processing to unrelated trials (i.e., slower RTs). This "cost" increases with strategic processing and thus provides a measure of the amount of bias operating within a condition. Under both high- and low-expectancy conditions, Goldinger's data show increasing RTs for unrelated trials as a function of the trials' temporal position. Thus, strategic processing is operative in both expectancy conditions. However, the "cost" is greater (a steeper increase in RTs as a function of position) in the highexpectancy condition than in the low-expectancy condition. As Goldinger admits, this result suggests, "The highexpectancy condition apparently created a stronger bias [than did the low-expectancy condition]" (p. 349). However, the extent of the variability observed in the high-expectancy condition coupled with the small number of observations per point prohibit strong conclusions from being drawn from this analysis.

\section{Time-Ordered Analysis of Hamburger and Slowiaczek (1996)}

Goldinger's (1999) primary argument centers on Hamburger and Slowiaczek's failure to eliminate bias in their low-expectancy condition, and he provides a timeordered analysis of his data to support this argument. A stronger test of this position would be a time-ordered analysis of the data from Hamburger and Slowiaczek (1996). To determine whether strategic processing was reduced in the low-expectancy condition of Hamburger and Slowiaczek, we subjected those data to the "timewindow" analysis proposed by Goldinger. Unrelated trials were grouped into five time windows: Trials $1-20$, Trials 21-40, Trials 41-60, Trials 61-80, and Trials 81-100. The mean RT for control trials within each time window was computed for each participant. Mean time window RTs were subjected to a 2 (expectancy) $\times 5$ (time window) analysis of variance. Expectancy was treated as a between-participants variable, and time window was treated as a within-participants variable. The degrees of freedom were modified by using the Huynh-Feldt correc- tion to adjust for violations of the sphericity assumption common in repeated measures designs (Jaccard \& Ackerman, 1985; Maxwell \& Avery, 1982). Results with $p<$ .05 were considered significant and will be reported. All results reported are statistically significant. Mean RTs for each time window and expectancy are shown in Table 1.

Inspection of the means in Table 1 shows longer RTs especially in the later trials for the high-expectancy condition in comparison with the low-expectancy condition. The time-ordered analysis revealed a main effect of expectancy $\left[F(1,130)=30.17, M S_{\mathrm{e}}=55,571\right]$ that reflects the longer RTs in the high-expectancy condition $(1,029 \mathrm{msec})$ than in the low-expectancy condition $(924 \mathrm{msec})$. Also, a main effect of time window was found $[F(4,494)=$ $\left.25.8, M S_{\mathrm{e}}=3,294\right]$ reflecting increasing RTs over the course of the experiment. Most importantly, the interaction was significant $\left[F(4,494)=2.84, M S_{\mathrm{e}}=3,294\right]$, indicating that a greater "cost" was observed in the highexpectancy condition.

To provide a more powerful analysis of increasing "costs" within each expectancy condition, a comparison of the slopes of lines regressing RTs for unrelated trials onto trial number for each participant was conducted. Because this analysis does not falsely attenuate variance by collapsing data across a time window, it should provide a more powerful statistical test. Regression equations predicting RTs on unrelated trials ( 25 trials per participant in the high-expectancy condition and 77 trials per participant in the low-expectancy condition) from trial number (i.e., temporal position in the experiment) were computed for each participant. The mean slopes of the equations for each expectancy were then compared in an independent groups $t$ test. This analysis revealed a significantly steeper slope in the high-expectancy condition (mean slope 1.19) than in the low-expectancy condition [mean slope, $.60 ; t(130)=4.96, S E=.119$ ]. Thus, "costs" increased more over time in the high-expectancy condition than in the low-expectancy condition. This result suggests that biases were reduced in the low-expectancy condition relative to the high-expectancy condition.

\section{Conclusions}

The significant interaction between expectancy and the time window in the time-ordered analyses demonstrates that lowering the PRP and shortening the ISI for the lowexpectancy condition did reduce strategic processing. Further evidence that biases were weaker in the lowexpectancy condition is provided by the finding of a significant difference between the slopes in the high- and low-expectancy conditions. This reduction in strategic

Table 1

Mean Response Times (in Milliseconds) for Each Time Window by Expectancy Condition

\begin{tabular}{cccccc}
\hline & \multicolumn{5}{c}{ Time Window } \\
\cline { 2 - 6 } Expectancy & Trials 1-20 & Trials 21-40 & Trials 41-60 & Trials 61-80 & Trials 81-100 \\
\hline High & 981 & 1,014 & 1,026 & 1,064 & 1,058 \\
Low & 901 & 914 & 917 & 936 & 951 \\
\hline
\end{tabular}


processing diminishes low-similarity facilitation and strengthens high-similarity interference, as was demonstrated by the priming by expectancy condition interaction observed by Hamburger and Slowiaczek (1996) and Goldinger (1999) in the initial analyses examining phonological priming in the RT data. Taken together, the results of these different analyses do support Goldinger's (1999; Goldinger et al., 1992) position that low-similarity facilitation is the product of a bias. However, the data do not support the idea that high-similarity interference is a product of the same bias. High-similarity interference is stronger, and evidence of bias is weaker, under lowexpectancy conditions. It is unlikely that a strategic effect would increase when the likelihood of strategic processing is decreased. Furthermore, the fact that high-similarity interference is dissociated from low-similarity facilitation suggests that the two effects have separate sources. The influence of prime lexicality on high-similarity interference suggests that this effect may be lexical in nature.

As Goldinger (1999) points out, the study of top-down processes in auditory word recognition deserves attention. Researchers must continue to ascertain the role of biases in the results of their research. However, the goal of language research is to understand the processes used in every day situations. In order to achieve that goal, priming data must be closely scrutinized to separate effects that reflect the normal processes of auditory word recognition and the unusual biases that result from the demands of an experiment. These processes can be separated by identifying dissociated effects and examining the changes in those effects when strategic processing is reduced. Therefore, it is not yet time to reconsider the "quest for 'pure' priming" (Goldinger, 1999, p. 350).

\section{REFERENCES}

Goldinger, S. D. (1999). Only the Shadower knows: Comment on Hamburger and Slowiaczek (1996). Psychonomic Bulletin \& Review, 6, 347-351.

Goldinger, S. D., Luce, P. A., Pisoni, D. B., \& Marcario, J. K. (1992). Form-based priming in spoken word recognition: The roles of competition and bias. Journal of Experimental Psychology: Learning, Memory, \& Cognition, 18, $1211-1238$.

Hamburger, M., \& SlowiaCzeK, L. M. (1996). Phonological priming reflects lexical competition. Psychonomic Bulletin \& Review, 3, 520-525.

JACCARD, J., \& ACKERMAN, L. (1985). Repeated measures analysis of means in clinical research. Journal of Consulting \& Clinical Psychologv, 53, 426-428.

MAXWELL, S. E., \& AVERY, R. (1982). Small sample profile analysis with many variables. Psychological Bulletin, 92, 778-785.

NEELY, J. H. (1977). Semantic priming and retrieval from lexical memory: Roles of inhibitionless spreading activation and limited-capacity attention. Journal of Experimental Psychology: General, 106, 226-254.

NEELY, J. H. (1991). Semantic priming effects in visual word recognition: A selective review of current findings and theories. In D. Besner \& G. Humphreys (Eds.), Basic processes in reading: Visual word recognition (pp. 264-336). Hillsdale, NJ: Erlbaum.

NEELY, J. H., \& KeEFE, D. E. (1989). Semantic effects on visual word processing: A hybrid prospective/retrospective processing theory. In G. H. Bower (Ed.), The psychology of learning and motivation: Advances in research and theory (Vol. 24, pp. 207-248). New York: Academic Press.

PosNer, M. I., \& SNYDER, C. (1975). Facilitation and inhibition in the processing of signals. In P. M. A. Rabbit \& S. Dornic (Eds.), Attention and performance $V$ (pp. 669-681). New York: Academic Press.

Slowiaczek, L. M., \& Hamburger, M. (1992). Prelexical facilitation and lexical inhibition in auditory word recognition. Journal of Experimental Psychology: Learning, Memory, \& Cognition, 18, 1239-1250.

Stone, M., JoAquim, S. G., \& Gabrieli, J. D. E. (1995). Anchoring effects in speed of word processing. Paper presented at the 36th Annual Meeting of the Psychonomic Society, Los Angeles.

(Manuscript received May 11, 1998; revision accepted for publication March 2, 1999.) 